\title{
Lamivudina por tempo prolongado no tratamento da hepatite B crônica no Estado de Mato Grosso
}

\author{
Long-term use of lamivudine for treating chronic hepatitis B \\ in the State of Mato Grosso
}

\author{
Francisco José Dutra Souto ${ }^{1}$, Ana Carolina da Silva Pirajá ${ }^{1}$, Graciana Soares da Silva ${ }^{1}$, \\ Marcelle Bottecchia ${ }^{2}$ e Selma Andrade Gomes ${ }^{2}$
}

\begin{abstract}
RESUMO
Para avaliar resultados do tratamento da hepatite B crônica com lamivudina, 100mg ou 150mg diários, foram acompanhados 34 pacientes em um serviço em Cuiabá, Mato Grosso. Entre os 34, 21 (62\%), eram cirróticos e 24 (70\%) HBeAg positivos. Genótipo viral foi determinado em 18, sendo predominante o genótipo A (12). O acompanhamento teve mediana de 27 meses (7 a 64). Do total, 23 (67\%) apresentaram resposta bioquímica entre dois e 24 meses de tratamento. Dos 24 pacientes com positividade para o HBeAg, 13 (54\%) apresentaram negativação do HBeAg durante o acompanhamento. Entre os anti-HBe positivos, 70\% tiveram normalização das aminotransferases. Quatorze (41\%) não apresentaram resposta bioquímica ou sorológica de início ou apresentaram breakthrough. Em seis dos que não responderam, foram encontradas as mutações L180M e M204V. Quatro pacientes faleceram após pelo menos 21 meses de lamivudina e três cirróticos desenvolveram hepatocarcinoma após 24 meses. A partir do terceiro ano surgiram complicações, como hepatocarcinoma ou hemorragia digestiva. Os presentes achados sugerem que resposta precoce ao tratamento com lamivudina pode estar associada a um melhor controle da hepatite B crônica.
\end{abstract}

Palavras-chaves: Virus da hepatite B. Tratamento. Lamivudina. Agentes antivirais.

\begin{abstract}
To assess the results from lamivudine treatment (100 $\mathrm{mg}$ or $150 \mathrm{mg}$ ) for chronic hepatitis $B, 34$ patients were followed at a clinic in Cuiabá, Mato Grosso, Central Brazil. Among them, 21 (62\%) had liver cirrbosis and 24 (70\%) were HBeAg-positive. The viral genotype was determined for 18 patients, among whom genotype A was the most prevalent (12). The median follow-up was 27 months (range from 7 to 64 months). Among the total, 23 (67\%) presented a biochemical response after 2 to 24 months of treatment. Among the 24 HBeAg-positive subjects, 13 (54\%) became HBeAg-negative during the follow-up. Among the anti-HBe-positive patients, 70\% obtained normalization of aminotransferase levels. Fourteen (41\%) did not present any initial biochemical or serological response or presented breakthrough. The L180M and M204V mutations were found in six of the non-responders. Four patients died after at least 21 months of lamivudine and three patients with liver cirrhosis developed liver cancer after 24 months. From the third year onwards, complications such as digestive system hemorrhage or liver cancer started to emerge. The present findings suggest that an early response to lamivudine treatment may be associated with better control over chronic hepatitis $B$.
\end{abstract}

Key-words: Hepatitis B virus. Treatment. Lamivudine. Antiviral agents.

A infecção crônica pelo vírus da hepatite B (VHB) é uma doença complexa que pode apresentar diferentes desfechos. É um importante problema de saúde pública mundial, podendo evoluir para cirrose hepática e carcinoma hepatocelular ${ }^{31}$. Por muitos anos, não se contou com qualquer tratamento, mas a partir dos anos 1990, com o emprego do interferon alfa, passou a ser possível alterar a história natural da doença ${ }^{29}$. Logo em seguida, notouse que a lamivudina (3TC), um análogo núcleosídico, diminuía a replicação do VHB e também podia interferir na evolução da doença ${ }^{6}$. Em que pese $o$ fato de apenas uma parcela dos indivíduos

1. Núcleo de Estudos de Doenças Infecciosas e Tropicais de Mato Grosso, Faculdade de Ciências Médicas, Universidade Federal de Mato Grosso, Cuiabá MT. 2. Laboratório de Virologia Molecular, Departamento de Virologia, Instituto Oswaldo Cruz, Rio de Janeiro, RJ.

Projeto financiado pelo CNPq.

Endereço para correspondência: Dr. Francisco José Dutra Souto. Av. Amarílio de Almeida 215/601, 78010-060 Cuiabá, MT.

Telefax: 5565 3624-7238

e-mail: fsouto@terra.com.br

Recebido em 08/05/2006

Aceito em 17/1/2007 
responde ao tratamento, estudos de seguimento comprovaram que ambos medicamentos diminuem a atividade inflamatória hepática; deprimem a replicação viral, aumentando a taxa de soroconversão do antígeno e (HBeAg) para o anticorpo correspondente (anti-HBe), e diminuem a carga viral, melhorando a histologia hepática ${ }^{10} 2228{ }^{30}$. Além disso, esses estudos demonstraram que a sobrevida sem complicações (descompensação de cirrose e surgimento de carcinoma hepatocelular) era maior nos grupos tratados quando comparados aos indivíduos que usaram placebo.

Apesar de discreta superioridade dos resultados do interferon alfa sobre os da lamivudina ${ }^{14}$, a toxicidade do primeiro, seu elevado índice de efeitos colaterais e necessidade de aplicação parenteral fizeram com que a lamivudina, de uso oral e com baixa frequiência de para-efeitos, passasse a ser largamente administrada em todo o mundo, inclusive em pacientes com insuficiência hepática, coisa impossível com o interferon alfa. Alguns pacientes com insuficiência hepática importante deixaram de necessitar de transplante hepático, graças à lamivudina ${ }^{28}$. Todavia, embora a lamivudina possa reduzir a carga viral do VHB rapidamente, tratamento prolongado pode ser necessário para se atingir os desfechos esperados ${ }^{12}$. Isso trouxe como complicação, a emergência de cepas resistentes à lamivudina (mutantes YMDD), que pode atingir 60\% dos pacientes após três anos de tratamento ${ }^{515}$. 0 advento das mutações que conferem resistência à lamivudina costuma recrudescer a hepatite B crônica, trazendo de volta o risco das complicações e, por vezes, produzindo graves flares de atividade necro-inflamatória hepática? .

Recentemente, novos análogos nucleot(s)ídicos, como o adefovir dipivoxil e o entecavir, foram desenvolvidos e aprovados para uso clínico ${ }^{316}$. Tais medicações têm demonstrado menores índices de cepas resistentes e a mesma segurança que a lamivudina. No entanto, essas novas drogas são muito caras e não há consenso ainda se devem ser usadas como terapia inicial ou após falha terapêutica de um dos agentes usados há mais tempo ${ }^{23}$. Outro fato singular no tratamento da hepatite B crônica é que, diferente de outras doenças infecciosas, a associação de agentes não tem mostrado maior supressão da quantificação viral, quando comparado ao uso das drogas isoladas ${ }^{1314}$.

Nos últimos anos, vários pesquisadores brasileiros têm tomado parte de ensaios clínicos multicêntricos internacionais sobre 0 tratamento das hepatites crônicas virais. No entanto, estes estudos costumam ter duração limitada a 12 meses de tratamento, com acompanhamento posterior de mais seis meses ${ }^{172122}$. Em nosso meio, são escassos os relatos sobre o uso prolongado de antivirais para hepatite B crônica. Porém, tais estudos são importantes para auxiliar na decisão sobre políticas públicas no tratamento da doença. 0 presente relato descreve a experiência com pacientes em tratamento com lamivudina por tempo prolongado no ambulatório de Hepatologia do Hospital da Universidade Federal de Mato Grosso (UFMT), em Cuiabá, que funciona como referência para a região.

\section{MATERIAL E MÉTODOS}

Este é um estudo de seguimento, aberto, iniciado em 1999 no ambulatório de Hepatologia do Hospital Universitário Júlio Muller (UFMT), Cuiabá, Mato Grosso. Também foram incluídos pacientes de clínica privada, acompanhados pela mesma equipe. Foram considerados para tratamento de hepatite B crônica, indivíduos com infecção pelo VHB documentada há pelo menos seis meses, que apresentassem um ou mais dos seguintes indícios de atividade necro-inflamatória hepática: i) elevação de aminotransferases na presença do HBeAg; ii) elevação de aminotransferases na ausência do HBeAg, mas com carga viral superior ou igual a $10^{5}$ cópias virais por $\mathrm{mL}$; iii) indivíduos com aminotransferases normais, mas com HBeAg positivo ou carga viral superior ou igual a $10^{4}$ cópias por $\mathrm{mL}$, que apresentassem sinais de cirrose. Foram incluídos indivíduos que não haviam usado lamivudina previamente, mas também indivíduos que não haviam respondido a tratamento com interferon alfa por quatro a seis meses.

Os pacientes que concordaram em participar receberam prescrição de dose diária de lamivudina de $100 \mathrm{mg}$ ou $150 \mathrm{mg}$, de acordo com o medicamento que estava disponível para distribuição na época pelo SUS local.

Os pacientes foram seguidos periodicamente com exame clínico, dosagem de aminotransferases e pesquisa do HBeAg e anti-HBe por método imuno-enzimático.

A frequiência do acompanhamento variou entre os indivíduos, dependendo de suas condições sócio-econômicas e particulares de comparecer ao serviço. A maioria dos pacientes negativos para o HBeAg foi submetida à quantificação do DNA do VHB por testes comerciais (Amplicor ${ }^{\circledR}$ HBV Monitor, Roche Diagnostic) com o fim de confirmar níveis replicativos do VHB na ausência do HBeAg ou após a normalização das aminotransferases.

Para determinação de mutações de resistência à lamivudina, o DNA do VHB foi extraído por fenol-clorofórmio e a região $S$ seqüenciada como descrito anteriormente ${ }^{1}$. Brevemente, a região pré-S/S do genoma viral foi amplificada por PCR em reação semi-nested, utilizando-se no primeiro ciclo de amplificação, o oligonucleotídeo senso C1 (5'-CTGTGGAGTTACTCTCGTTTTTGC-3;, posição de nt 1935-1958), e uma mistura de oligonucleotídeos anti-senso S2 (5-GGGTTTAAATGTATACCCAAAGA-3’, 819-841) e S22 (5-GTATTTAAATGGATACCCACAGA-3’, 819-841). 0 segundo ciclo de amplificação foi realizado com os oligonucleotídeos PS1 (5-CCATATTCTTGGGAACAAGA-3`, 2826-2845) e S2+ S22.

Os produtos da PCR foram submetidos à eletroforese preparativa em géis de agarose, excisados dos géis e purificados através do kit comercial QIAquick Gel Extraction Kit (QIAGEN). Os produtos da PCR purificados foram preparados para o seqüenciamento de nucleotídeos utilizando-se o kit comercial Big Dye Terminator 3.1 Cycle Sequencing Kit (Applied Biosystems). Uma série de oligonucleotídeos senso e anti-senso foram utilizados para sequenciamento de ambas as fitas da região $S$ do VHB.

A leitura automática das seqüências foram feitas com 0 equipamento ABI 3730 DNA Analyzer (Applied Biosystems). Para construção das árvores filogenéticas, as seqüências nucleotídicas foram alinhadas a partir do códon de iniciação da região $S$. A tradução das sequiências em aminoácidos, para fase de leitura da polimerase viral, possibilitou a análise das mutações de resistência à lamivudina. 
A determinação dos genótipos do VHB foi feita pela técnica de restriction fragment lenght polymorphism (RFLP), conforme descrito anteriormente ${ }^{1}$.

Todos os participantes assinaram consentimento pósesclarecimento. 0 protocolo foi submetido e aprovado pelo Comitê de Ética em Pesquisa em Seres Humanos do Hospital Universitário Júlio Muller (23/1999). Não foi criado grupo controle sem tratamento porque, à luz dos conhecimentos atuais, seria antiético deixar tais pacientes seguindo a evolução natural da infecção. Foram avaliadas a resposta bioquímica (normalização dos níveis de aminotransferases), a resposta virológica nos pacientes HBeag positivos (negativação do HBeAg e/ou soroconversão HBeAg/antiHBe) e a evolução clínica, incluindo a classificação de Child-Pugh em pacientes cirróticos. Também foram descritas as frequiências de complicações, como não resposta a tratamento, progressão da doença hepática, e surgimento de carcinoma hepatocelular. A incidência de negativação do HBeAg em relação ao tempo de tratamento e a incidência de resposta bioquímica foi demonstrada por meio do método de Kaplan-Meier, utilizando-se o programa estatístico SPSS 13.0 ( SPSS Inc., Chicago, EUA, 2004).

\section{RESULTADOS}

A partir de janeiro de 1999, 41 pacientes foram incluídos no estudo. Ainda no início do seguimento, sete foram excluídos por abandono precoce do acompanhamento ambulatorial. Dos 34 restantes, 25 (73\%) eram homens. A mediana e a média da idade foram de 42 anos, variando de 12 a 76. Dezessete (50\%) residiam na região metropolitana da capital do estado. A maioria era nascida nas regiões Sudeste e Sul do país (68\%). Vinte e um (62\%) já tinham sinais clínicos, laboratoriais ou histopatológicos de cirrose, sendo 10 (29\%) Child A, sete (21\%) Child B e 4 (12\%) Child C. Dos 13 sem cirrose, seis (18\%) já haviam sido tratados com interferon-alfa. Ao começar o tratamento com lamivudina, 32 (94\%) tinham aminotransferases elevadas (ALT e/ou AST) e 24 (70\%) eram HBeAg-positivos. Os dois (6\%) que não tinham aumento das aminotransferases, eram uma mulher de 21 anos, HBeAg positiva, com $10^{7}$ cópias/mL de DNA do VHB e plaquetopenia de 60.000 , razão pela qual não foi submetida à biopsia hepática; e um homem de 38 anos, HBeAg positivo, com biopsia evidenciando cirrose hepática. A estatística descritiva e as características individuais dos pacientes são apresentadas nas Tabelas 1 e 2, respectivamente.

Foi possível mensurar a carga viral antes do início do tratamento em oito dos dez pacientes anti-HBe positivos, que revelou mediana de $10^{6}$, variando de $10^{4}$ a $10^{8}$ cópias $/ \mathrm{mL}$. Quanto aos pacientes HBeAg positivos, a carga viral não foi considerada essencial para a indicação do tratamento. Entre os 24 pacientes com esse perfil sorológico, 12 tiveram medida da carga viral antes do início do tratamento, com mediana de $10^{7}$ e variação de $10^{5}$ a $10^{8}$ cópias $/ \mathrm{mL}$.

Para efeito deste relato, o acompanhamento dos pacientes foi interrompido em junho de 2005, sendo que a mediana do tempo de tratamento foi de 26 meses, variando de 7 a 64 . Após o início do tratamento, houve queda das aminotransferases na maioria dos pacientes. Após 24 meses, restavam 16 pacientes ainda em acompanhamento. Os outros 18 tiveram perda do seguimento ou tiveram seu tratamento interrompido por terem atingido resposta bioquímica (normalização das aminotransferases) e/ou virológica (negativação do HBeAg).

Do total, 23 (67\%) pacientes apresentaram normalização das aminotransferases. Vinte deles dentro dos primeiros 24 meses de tratamento (Figura 1). Os onze pacientes restantes apresentaram diminuição dos níveis das aminotransferases, mas sem normalização. Quatro pacientes, que responderam inicialmente e tiveram o tratamento interrompido, voltaram a apresentar elevação de aminotransferases. A reintrodução da medicação não voltou a mostrar o mesmo efeito. Deste modo, 15 (44\%) pacientes apresentaram falha terapêutica inicial ou tardia à lamivudina. Foi realizada pesquisa de mutações do gen da polimerase relacionadas à resistência à lamivudina em oito desses 15. Em seis foi confirmada a presença tanto da mutação L180M, quanto da M204V. Nos dois restantes, não foram detectadas mutações de resistência à lamivudina.

Dos 24 pacientes HBeAg positivos, 13 (54\%) apresentaram negativação do HBeAg durante o acompanhamento, mas a soroconversão para anti-HBe só foi registrada em cinco (21\%) deles. A mediana do tempo para negativação do HBeAg foi de 12 meses, variando de 2 a 41 meses. Os pacientes que apresentaram soroconversão HBeAg para anti-HBe tiveram o tratamento interrompido, caso também apresentassem resposta bioquímica. A Figura 2 apresenta a evolução da negativação do HBeAg ao longo do tempo. Os 13 pacientes que negativaram o HBeAg ou apresentaram soroconversão para anti-HBe representaram uma incidência acumulada de 29 desses eventos por 100 pessoasano. A negativação do HBeAg ocorreu antes de 24 meses em

Tabela 1- Característica dos pacientes com infecção crônica pelo vírus da hepatite $B$, com atividade inflamatória hepática, acompanhados entre 1999 e 2005, no Hospital Júlio Muller, Cuiabá, Mato Grosso.

\begin{tabular}{|c|c|c|c|c|c|c|}
\hline \multirow[t]{2}{*}{ Características } & \multicolumn{2}{|c|}{ Total } & \multicolumn{2}{|c|}{$\mathrm{HBeAg}+$} & \multicolumn{2}{|c|}{ Anti-HBe + } \\
\hline & $\mathrm{n}^{0}$ & $\%$ & $\mathrm{n}^{0}$ & $\%$ & $\mathrm{n}^{0}$ & $\%$ \\
\hline Número & 34 & 100,0 & 24 & 71,0 & 10 & 29,0 \\
\hline Sexo masculino & 25 & 73,0 & 17 & 68,0 & 7 & 78,0 \\
\hline Idade média (erro padrão) & 42 & 3,0 & 39 & 16,0 & 49 & 14,0 \\
\hline \multicolumn{7}{|l|}{ Região brasileira de origem: } \\
\hline Mato Grosso & 7 & 21,0 & 6 & 85,0 & 1 & 15,0 \\
\hline sudeste & 14 & 41,0 & 10 & 71,0 & 4 & 29,0 \\
\hline sul & 9 & 26,0 & 4 & 44,0 & 5 & 56,0 \\
\hline nordeste & 4 & 12,0 & 4 & 100,0 & & 0 \\
\hline Mediana idade (quartis 25,75 ) & 42 & 27,56 & 39 & 25,43 & 48 & 41,58 \\
\hline Cirrose & 21 & 62,0 & 13 & 54,0 & 8 & 80,0 \\
\hline \multicolumn{7}{|l|}{ Child-Pugh } \\
\hline A & 10 & 48,0 & 5 & 38,0 & 5 & 62,0 \\
\hline B & 7 & 33,0 & 5 & 38,0 & 2 & 25,0 \\
\hline C & 4 & 19,0 & 3 & 24,0 & 1 & 12,0 \\
\hline ALT e/ou AST elevadas & 32 & 94,0 & 22 & 92,0 & 10 & 100,0 \\
\hline Média ALT/LMN* & & 1,9 & & 2,2 & & 1,4 \\
\hline Média AST/LMN* & & 2,1 & & 2,2 & & 2,0 \\
\hline
\end{tabular}

* LMN: Limite máximo da normalidade. 
Tabela 2 - Características e evolução de 34 pacientes com hepatite B crônica e resultados de seu acompanhamento, no Ambulatório de Hepatologia do Hospital Júlio Muller, Cuiabá, Mato Grosso.

\begin{tabular}{|c|c|c|c|c|c|c|c|c|c|c|c|c|c|}
\hline Paciente* & HBe Ag & Id & Sexo & $\begin{array}{c}\text { Raz } \\
\text { ALT inic }\end{array}$ & $\mathrm{CV}$ inic & $\begin{array}{l}\text { Cirrose } \\
\text { (Child) }\end{array}$ & Genótipo & Tempo lam & $\begin{array}{c}\text { Resp } \\
\text { bioq inic. }\end{array}$ & $\begin{array}{c}\text { Neg } \\
\text { HBe Ag }\end{array}$ & $\begin{array}{c}\text { Tempo HBeAg } \\
\text { negat }\end{array}$ & Falha lam & Mut ymdd \\
\hline 1 & + & 12 & $\mathrm{M}$ & 1,9 & $10^{7}$ & - & $\mathrm{Aa}$ & 24 & - & + & 21 & + & + \\
\hline 2 & + & 38 & M & 0,7 & - & A & $\mathrm{F}$ & 64 & - & + & 3 & + & + \\
\hline 3 & + & 47 & M & 1,3 & - & B & $\mathrm{Aa}$ & 28 & + & + & 12 & - & \\
\hline 4 & + & 58 & $\mathrm{~F}$ & 1,2 & - & B & - & 36 & - & - & & + & \\
\hline 5 & + & 37 & M & 2,4 & $10^{5}$ & $\mathrm{C}$ & $\mathrm{F}$ & 23 & + & + & 6 & - & \\
\hline 6 & + & 41 & M & 1,3 & $10^{5}$ & C & $\mathrm{F}$ & 13 & + & + & 7 & - & \\
\hline 7 & + & 53 & M & 1,8 & - & A & $\mathrm{Ae}$ & 59 & - & - & & + & + \\
\hline 8 & + & 43 & M & 1,2 & - & B & - & 47 & - & + & 41 & + & \\
\hline 9 & + & 59 & M & 1,1 & - & A & - & 44 & + & + & 35 & - & \\
\hline 10 & + & 27 & M & 1,5 & $10^{6}$ & - & - & 19 & + & + & 12 & - & \\
\hline 11 & + & 57 & $\mathrm{~F}$ & 1,4 & - & A & - & 51 & + & - & & - & \\
\hline 12 & + & 25 & $\mathrm{~F}$ & 1,5 & $10^{7}$ & - & C & 51 & - & - & & + & + \\
\hline 13 & + & 47 & M & 2,0 & $10^{8}$ & - & $\mathrm{Aa}$ & 33 & - & - & & + & + \\
\hline 14 & + & 43 & M & 1,8 & $10^{5}$ & C & - & 45 & + & + & 14 & + & \\
\hline 15 & + & 56 & M & 3,6 & $10^{5}$ & B & $\mathrm{Aa}$ & 26 & + & - & & + & - \\
\hline 16 & + & 53 & M & 2,4 & $10^{5}$ & - & $\mathrm{Aa}$ & 12 & + & - & & - & - \\
\hline 17 & + & 71 & $\mathrm{~F}$ & 1,4 & - & B & $\mathrm{Aa}$ & 15 & + & + & 2 & - & \\
\hline 18 & + & 12 & $\mathrm{~F}$ & 4,9 & - & - & - & 34 & + & + & 22 & - & \\
\hline 19 & + & 33 & M & 2,2 & - & A & $\mathrm{Ae}$ & 48 & - & + & 32 & + & + \\
\hline 20 & + & 25 & M & 3,5 & - & - & - & 22 & + & - & & - & \\
\hline 21 & + & 21 & $\mathrm{~F}$ & 0,9 & $10^{7}$ & - & $\mathrm{Aa}$ & 12 & + & - & & - & - \\
\hline 22 & + & 24 & M & 7,4 & $10^{7}$ & - & - & 22 & + & - & & + & \\
\hline 23 & + & 25 & $\mathrm{~F}$ & 0,8 & $10^{7}$ & - & D & 7 & - & - & 7 & + & - \\
\hline 24 & + & 21 & M & 3,6 & - & - & $\mathrm{Aa}$ & 12 & + & + & 12 & - & \\
\hline 25 & - & 57 & $\mathrm{~F}$ & 1,3 & $10^{4}$ & A & - & 21 & + & & & + & \\
\hline 26 & - & 58 & $\mathrm{~F}$ & 1,1 & $10^{6}$ & B & - & 26 & + & & & - & \\
\hline 27 & - & 34 & M & 1,9 & - & B & - & 15 & + & & & - & \\
\hline 28 & - & 52 & M & 2,1 & $10^{6}$ & A & - & 29 & - & & & + & \\
\hline 29 & - & 76 & M & 2,4 & $10^{6}$ & A & $\mathrm{Ae}$ & 34 & + & & & - & - \\
\hline 30 & - & 41 & M & 2,3 & - & - & - & 37 & + & & & - & \\
\hline 31 & - & 63 & M & 1,5 & $10^{8}$ & A & D & 20 & + & & & - & - \\
\hline 32 & - & 44 & M & 1,2 & $10^{7}$ & $\mathrm{C}$ & - & 7 & - & & & + & \\
\hline 33 & - & 41 & M & 1,8 & $10^{5}$ & - & - & 43 & + & & & - & \\
\hline 34 & - & 28 & M & 1,8 & $10^{4}$ & A & $\mathrm{Aa}$ & 13 & + & & & - & - \\
\hline
\end{tabular}

*número do paciente; Id: idade no início do tratamento; Raz ALT inic: razão entre ALT inicial e valor máximo normal; CV inic: carga viral inicial; tempo lam: tempo de uso de lamivudina durante o seguimento; Resp. bioq. Inic: resposta bioquímica inicial; Neg. HBeAg: negativação do HBeAg; Tempo HBeAg negat: tempo de uso de lamivudina até ocorrer negativação do HBeAg; Falha lam: pacientes que tiveram falha da lamivudina (resistência primária ou breakthrough); Mut ymdd: resistência à lamivudina.

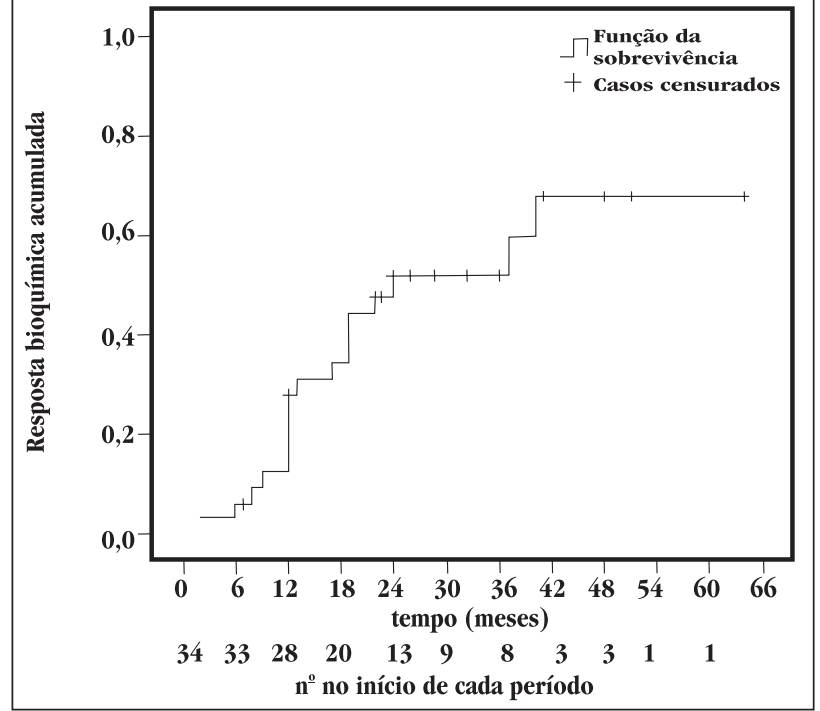

Figura 1- Curva de probabilidade (método de Kaplan-Meier) de resposta bioquímica no decorrer do tratamento com lamivudina em 34 pacientes com hepatite B crônica, Cuiabá, Mato Grosso.

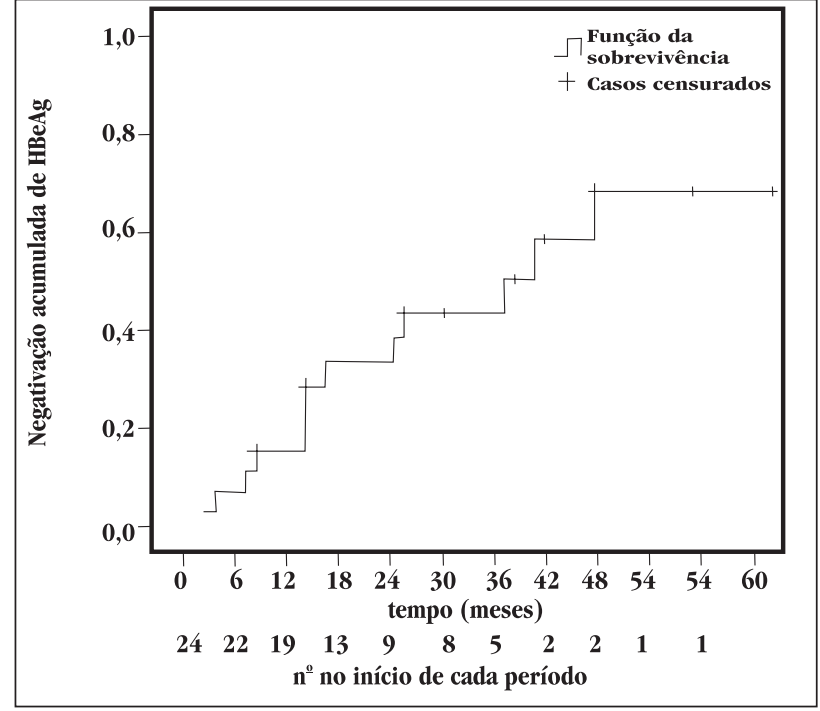

Figura 2 - Curva de probabilidade (método de Kaplan-Meier) de negativação do HBeAg no decorrer do tratamento com lamivudina em 24 pacientes com hepatite B crônica HBeAg-positivos, Cuiabá, Mato Grosso. 
dez (77\%) dos 13. Nos três restantes a soroconversão ocorreu mais tarde (32, 35 e 41 meses).

Entre os dez pacientes anti-HBe positivos, sete tiveram normalização das aminotransferases entre 12 e 24 meses, mas não tiveram o tratamento interrompido. Um deles voltou, meses depois, a apresentar elevação das aminotransferases, somando então quatro falhas entre esses pacientes.

Após seis meses de tratamento, nenhum dos 21 pacientes cirróticos piorou a classificação de Child-Pugh, havendo melhora em seis (de B para A em 4, e C para B em 2). Aos 12 meses, o acompanhamento de 19 cirróticos, mostrou piora em um (de B para C) e melhora em dois (de B para A) e aos 24 meses, nenhum dos dez cirróticos ainda em acompanhamento apresentou piora. No entanto, após 24 meses, três pacientes cirróticos, sendo um HBeAg-positivo e dois HBeAg-negativos ao inicio do tratamento, desenvolveram hepatocarcinoma. Quatro pacientes cirróticos faleceram após pelo menos 21 meses de lamivudina. Dois faleceram após desenvolvimento de hepatocarcinoma, um por sangramento de varizes esofagianas e outro por peritonite bacteriana espontânea. Dois (6\%) pacientes, que eram HBeAgnegativos no início, e que receberam lamivudina por 15 e 37 meses, apresentaram soroconversão $\mathrm{HBsAg}$ /anti-HBs. 0 primeiro deles, dois anos após ter deixado de usar lamivudina.

Foi possível determinar o genótipo do VHB, em 18 dos 34 pacientes. Foram encontrados os genótipos A em 12 indivíduos, o F em três, o D em dois, e o C, em uma jovem descendente de japoneses oriunda do estado de São Paulo. Dos 12 genótipos A, nove eram do subtipo africano e três do europeu (Tabela 2).

\section{DISCUSSÃo}

O tratamento da hepatite B crônica é sujeito a muitas variáveis e apresenta bons resultados apenas em uma parcela dos pacientes acometidos. No Brasil, nos defrontamos com dificuldades no monitoramento dos pacientes infectados e com número reduzido de opções terapêuticas acessíveis para a maioria da população. Este relato apresenta a experiência de um Serviço de Hepatologia de pequeno porte no interior do Brasil, que enfrenta as dificuldades vividas pela maioria dos profissionais médicos no país, tentando lidar com uma doença complexa em um cenário de carência de recursos.

A indicação para uso de lamivudina neste grupo se justificava porque a maioria era constituída de cirróticos, não podendo usar interferon alfa. Dentre os não-cirróticos, seis já haviam usado interferon alfa, sem sucesso. Os pacientes restantes optaram por receber lamivudina depois de esclarecidos sobre os efeitos colaterais do interferon alfa. Para alguns, lamivudina foi indicada por residirem no interior do estado, em locais distantes do nosso serviço e com menos recursos.

A maioria (67\%) dos pacientes apresentou resposta bioquímica à lamivudina, com normalização das aminotransferases. Isto ocorreu em dez indivíduos no primeiro ano e em outros dez no ano seguinte. Apenas três normalizaram as aminotransferases depois de 24 meses, indicando que, se não houver resposta nos dois primeiros anos, fica mais difícil alcançar esse objetivo depois disso.
O grupo em estudo foi constituído, em sua maioria, por pacientes HBeAg-positivos, provavelmente pela grande presença do genótipo $\mathrm{A}$, que desenvolve mutação pré-core menos freqüentemente ${ }^{19}$. Cerca de metade (54\%) dos pacientes HBeAg positivos apresentou negativação deste marcador com o uso da lamivudina, sendo que $77 \%$ dos que negativaram o HBeAg atingiram esse objetivo antes dos 24 meses, novamente sugerindo que a chance de resposta à lamivudina diminui depois deste período. No entanto, outros autores detectaram crescente proporção de resposta à lamivudina com a extensão do tratamento até quatro anos ${ }^{4}$.

Quando o desfecho avaliado foi a soroconversão do HBeAg para 0 anti-HBe, apenas cinco (15\%) pacientes tiveram esta evolução comprovada, semelhante ao descrito por Liaw e cols 15 em pacientes chineses após um ano de tratamento. Jonas e cols $^{12}$, estudando crianças menores de 17 anos, relataram 23\% de negativação do HBeAg com um ano de lamivudina. No presente estudo, sete (20\%) negativaram até o $12^{\circ}$ mês.

Entre os dez pacientes inicialmente HBeAg negativos, supostamente portadores de cepas com mutação pré-core, houve resposta bioquímica na maioria, o que está de acordo com outros autores que demonstraram que os pacientes com cepas mutantes anti-HBe positivas também se beneficiam do tratamento com lamivudina ${ }^{11} 2027$. No Brasil, outros autores também mostraram resultados favoráveis com a lamivudina em hepatite B crônica ${ }^{1721} 22$, embora alguns tivessem utilizado doses mais elevadas da droga, como usado na infecção pelo HIV ${ }^{22}$.

Apesar dos bons índices de resposta bioquímica e virológica iniciais aqui relatados, dez pacientes não mostraram resposta em nenhum momento e quatro que haviam respondido voltaram a apresentar aminotansferases elevadas, resultando em um total de $44 \%$ com evidências de resistência à droga. Esses dados são semelhantes aos referidos na literatura: 17 a $20 \%$ no primeiro ano de tratamento, $40 \%$ no segundo e, 51 a $67 \%$ no terceiro ${ }^{59}$. Coincidentemente, 0 índice de falha terapêutica com lamivudina em nosso estudo foi semelhante 14/38 (42\%) ao encontrado em outro estudo brasileiro ${ }^{17}$. Em seis desses pacientes foi possível documentar a presença de mutações de resistência no gen da polimerase.

Mato Grosso é uma região caracterizada por forte migração nas últimas décadas, o que influencia o perfil genotípico observado ${ }^{26}$. Embora a subamostra em que foi possível definir o genótipo fosse pequena (53\%), confirmou-se a presença de genótipos já descritos no Brasil ${ }^{218}$. 0 genótipo A foi o mais comum, provavelmente porque a maior parte da casuística era originária da região Sudeste do Brasil. 0 genótipo $\mathrm{C}$, mais freqüente na Ásia, apareceu em um descendente oriental, e o F, em pessoas oriundas da região Nordeste. No entanto, a presença do genótipo $\mathrm{D}$ foi muito pequena em relação à subtipagem do HBsAg correspondente (ayw2 e ayw3), descrita previamente em outros estudos realizados em Mato Grosso ${ }^{24} 25$. A explicação provável para essa diferença é que os pacientes aqui descritos têm origem diferente dos estudados em relatos anteriores, onde a quase totalidade era originária do Sul do país e residia na porção amazônica do estado de Mato Grosso. Na presente casuística, apenas 26\% eram nascidos na região Sul.

No grupo dos pacientes cirróticos $(60 \%)$ houve melhora na classificação de Child-Pugh no início do tratamento. Este achado 
reforça a impressão de que a lamivudina pode sesr útil para pacientes com cirrose descompensada em fila de transplante hepático. Porém, especialmente após 24 meses de seguimento, as complicações, como a peritonite espontânea, a hemorragia digestiva alta e 0 carcinoma hepatocelular, foram evidenciadas. Infelizmente, em nosso meio, o tempo de espera para o transplante hepático tem sido maior que dois anos, o que aumenta a chance destas complicações da cirrose hepática e da emergência de resistência à droga.

Curiosamente, dois (6\%) pacientes apresentaram tardiamente soroconversão de HBsAg para anti-HBs, efeito raramente descrito com o uso de lamivudina e mais observado, embora também incomum, durante 0 tratamento com interferon alf ${ }^{14}$. Lau e $\operatorname{cols}^{14}$ não registraram soroconversão do HBsAg no grupo que recebeu lamivudina, mas a droga foi administrada apenas por 12 meses, com seguimento posterior por mais seis meses. No nosso estudo, os dois casos que apresentaram soroconversão, o fizeram tardiamente. Um deles, dois anos após ter usado 15 meses de lamivudina, com sucesso. De fato, não se pode afirmar que o tratamento foi o determinante dessa soroconversão. Porém, é possível que, se a observação de Lau e cols ${ }^{14}$ fosse mais prolongada, talvez tivessem também percebido a ocorrência de soroconversão HBsAg-anti-HBs tardiamente.

Em suma, o tratamento da hepatite B com lamivudina, em um grupo com grande freqiência de pacientes HBeAg positivos e de cirróticos, apresentou resposta favorável em mais da metade dos pacientes, com melhora bioquímica e/ou virológica. No entanto, este efeito benéfico não foi mantido após 24 meses do início do tratamento, quando começaram a surgir complicações e morte. Este achado é compativel com o descrito por Furusyo e cols ${ }^{8}$, em que a taxa de reativação viral (breakthrough) foi tão mais freqüente quanto mais tardia havia ocorrido a resposta bioquímica ou virológica.

0 menor custo da medicação e sua grande tolerabilidade a tornam útil, especialmente no grupo de pacientes com cirrose, mesmo com o advento de novas medicações com melhor perfil de resistência, como o adefovir e o entecavir. Aparentemente, a lamivudina continuará tendo função no arsenal terapêutico contra o VHB nos próximos anos, pelo menos até que se esclareça melhor a estratégia ideal no controle dessa infecção.

\section{REFERÊNCIAS}

1. Araújo NM, Mello FCA, Yoshida CFT, Niel C, Gomes AS. High proportion of subgroup A`(genotype A) among Brazilian isolates of hepatitis B virus. Archives of Virology 149:1383-1395, 2004.

2. Carrilho FJ, Moraes CR, Pinho JR, Mello IM, Bertolini DA, Lemos MF, Moreira RC, Bassit LC, Cardoso RA, Ribeiro-dos-Santos G, da Silva LC. Hepatitis B virus infection in Haemodialysis Centres from Santa Catarina State, Southern Brazil. Predictive risk factors for infection and molecular epidemiology. BMC Public Health 27:4-13, 2004.

3. Chang TT, Gish RG, Hadziyannis SJ, Cianciara J, Rizzetto M, Schiff ER, Pastore G, Bacon BR, Poynard T, Joshi S, Klesczewski KS, Thiry A, Rose RE, Colonno RJ, Hindes RG; BEHoLD Study Group. A dose-ranging study of the efficacy and tolerability of entecavir in Lamivudine-refractory chronic hepatitis B patients. Gastroenterology 129:1198-1209, 2005.

4. Chang TT, Lai CL, Chien RN, Guan R, Lim SG, Lee CM, Ng KY, Nicholls GJ, Dent JC, Leung NW. Four years of lamivudine treatment in Chinese patients with chronic hepatitis B. Journal of Gastroenterology and Hepatology 19:1276-1282, 2004.
5. Conjeevaram HS, Lok AS. Managemente of Cronic Hepatitis B. Journal of Hepatology 38:\$90-\$103, 2003.

6. Dienstag JL, Perrillo RP, Schiff ER, Bartholomew M, Vicary C, Rubin M. A preliminary trial of lamivudine for chronic hepatitis B infection. New England Journal of Medicine 333:1657-1661, 1995.

7. European Association for the Study of the Liver. International Consensus Conference on Hepatitis B. Journal of Hepatology 38:533-540, 2003.

8. Furusyo N, Takeoka H, Toyoda K, Murata M, Tanabe Y, Kajiwara E, Shimono J, Masumoto A, Maruyama T, Nomura H, Nakamuta M, Takahashi K, Shimoda S, Azuma K, Sakai H, Hayashi J. Long-term lamivudine treatment for chronic hepatitis B in Japanese patients: A project of Kyushu University Liver Disease Study. World Journal of Gastroenterology 12:561-567, 2006.

9. Ganem D, Prince AM. Hepatitis B Viral Infection - Natural History and Clinical Consequences. New England Journal of Medicine 350:1118-1129, 2004.

10. Ikeda K, Saitoh S, Suzuki Y, Kobayashi M, Tsubota A, Koida I, Arase Y, Fukuda M, Chayama K, Murashima N, Kumada H. Disease progression and hepatocellular carcinogenesis in patients with chronic viral hepatitis: a prospective observation of 2215 patients. Journal of Hepatology 28: 930-938, 1998.

11. Jang JW, Bae SH, Choi JY, Kim CW, Han NI, Han JY, Choi SW, Yoon SK, Chung KW, Sun HS. Early virological response predicts outcome during extended lamivudine retreatment in patients with chronic hepatitis B who relapsed after initial HBeAg responses. Journal of Gastroenterology and Hepatology 21:384-391, 2006.

12. Jonas MM, Kelley DA, Mizerski J, Badia IB, Areias JA, Schwartz KB, Little NR, Greensnith MJ, Gardnes SD, Bell MS, Sokal EM. Clinical trial of lamivudine in children with chronic hepatitis B. New England Journal of Medicine 342:1706$1713,2002$.

13. Kim KM, Choi WB, Lim YS, Lee HC, Chung YH, Lee YS, Suh DJ. Adefovir dipivoxil alone or in combination with ongoing lamivudine in patients with decompensated liver disease and lamivudine-resistant hepatitis B virus. Journal of Korean Medical Science 20:821-828, 2005.

14. Lau GK, Piratvisuth T, Luo KX, Marcellin P, Thongsawat S, Cooksley G, Gane E, Fried MW, Chow WC, Paik SW, Chang WY, Berg T, Flisiak R, Mccloud P, Pluck N. Peginterferon Alfa-2a HBeAg-Positive Chronic Hepatitis B Study Group. Peginterferon Alfa-2a, lamivudine, and the combination for HbeAg-positive chronic hepatitis B. New England Journal of Medicine 352:2682-2695, 2005.

15. Liaw YF, Leung NWY, Chang TT, Guan R, Tai DI, Ng KY, Chien RN, Dent J, Roman L, Edmundson S, Lai CL. Effects of extended lamivudine therapy in Asian patients with chronic hepatitis B. Gastroenterology 119:172-180, 2000.

16. Marcellin P, Chang TT, Lim SG, Tong MJ, Sievert W, Shiffman ML, Jeffers L, Goodman Z, Wulfsohn MS, Xiong S, Fry J, Brosgart CL. Adefovir Dipivoxil 437 Study Group. Adefovir dipivoxil for the treatment of hepatitis B antigen-positive chronic hepatitis B. New England Journal of Medicine 348:808-816, 2003.

17. Nabuco LC, Villela-Nogueira CA, Perez RM, Quintaes R, Segadas-Soares JA, Brandão CE, Cardoso AC, Leite NC, Coelho HSM. Avaliação da resposta ao tratamento com lamivudina em pacientes com hepatite crônica B HBeAg positivo. Gastroenterologia e Endoscopia Digestiva 24 (supl 2):S13, 2005.

18. Paraná R, Almeida D. HBV epidemiology in Latin América. Journal of Clinical Virology 34(supl I):S130-S133, 2005.

19. Rodriguez-Frias F, Buti M, Jardi R, Cotrina M, Viladomiu L, Esteban R, Guardia $\mathrm{J}$. Hepatitis B vírus infection: precore mutants and its relation to viral genotypes and core mutations. Hepatology 22:1641-1647, 1995.

20. Santantonio T, Mazzola M, Iacovazzi T, Miglieta A, Guastadisegni A, Pastore G. Long-term follow-up of patients with anti-HBe/HBV DNA positive chronic hepatitis B treated for 12 months with lamivudine. Journal of Hepatology 32:300-306, 2000.

21. Silva LC, Fonseca LEP, Carrilho FJ, Alves VAF, Sitnik R, Pinho JRR. Predictive factors for response to lamivudine in chronic hepatitis B. Revista do Institito de Medicina Tropical de São Paulo 42:189-196, 2000.

22. Silva LC, Pinho JRR, Sitnik R, Fonseca LEP, Carrilho FJ. Efficacy and tolerability of long-term therapy using high lamivudine doses for the treatment of chronic hepatitis B. Journal of Gastroenterology 36:476-485, 2001.

23. Sociedade Brasileira de Hepatologia. Consenso sobre condutas nas hepatites B e C: Hepatite B. Gastroenterologia e Endoscopia Digestiva 24 (supl 1):S1-S8, 2005. 
24. Souto FJD, Fontes CJF, Gaspar AMC. Outbreak of hepatitis B virus in recent arrivals to the Brazilian Amazon. Journal of Medical Virology 56:4-9, 1998.

25. Souto FJD, Fontes CJF, Gaspar AMC, Lyra LGC. Hepatitis B virus infection in immigrants to the southern Brazilian Amazon. Transactions of Royal Society of Tropical Medicine and Hygiene 92:282-284, 1998.

26. Souto FJD, Fontes CJF, Oliveira SS, Yonamine F, dos Santos DRL, Gaspar AMC. Prevalência da hepatite B em área rural de município hiperendêmico na Amazônia mato-grossense: situação epidemiológica. Epidemiologia e Serviços de Saúde 13:93-102, 2004.

27. Tassopoulos NC, Volpes R, Pastore G, Heathcote J, ButiM, Goldin RD, Hawley S, Barber J, Condreay L, Gray DF, and the Lamivudine Precore Mutant Study Group. Efficacy of lamivudine in patients with hepatitis B e antigen-negative/hepatitis B virus DNA-positive (precore mutant) chronic hepatitis B. Hepatology 29:889-896, 1999
28. Villeneuve JP, Condreay LD, Willems B, Pomier-Layragues G, Fenyves D, Bilodeau M, Leduc R, Peltekian K, Wong FL, Margulies M, Heathcote EJ. Lamivudine treatment for decompensated cirrhosis resulting from chronic hepatitis $\mathrm{B}$. Hepatology 31:207-210, 2000.

29. Wong DK, Cheung AM, O'Rourke K, Naylor CD, Detsky AS, Heathcote J. Effect of alpha-interferon treatment in patients with hepatitis $B$ e antigen-positive chronic hepatitis B: A meta-analysis. Annals of Internal Medicine 119:312-323, 1993.

30. Yao FY, Terrault NA, Freise C, Maslow L, Bass NM. Lamivudine treatment is beneficial in patients with severely decompensated cirrhosis and actively replicating hepatitis $B$ infection awaiting liver transplantation: a comparative study using a matched, untreated cohort. Hepatology 34:411-416, 2001.

31. Zuckerman JN, Zuckerman AJ. The epidemiology of hepatitis. Clinical in Liver Diseases 3:179-188, 1999. 